\title{
Adsorption Separation of Arsenic(III) and Arsenic(V) using Functionalized Silica Gels
}

\author{
Elena V. Borodina ${ }^{a}$, Svetlana L. Didukh-Shadrina*a, \\ Vladimir N. Losev and Anatoly K. Trofimchuk ${ }^{\mathrm{b}}$ \\ ${ }^{a}$ Scientific research engineering center «Kristall» \\ Siberian Federal University \\ Krasnoyarsk, Russian Federation \\ ${ }^{b}$ Department of Inorganic Chemistry \\ Taras Shevchenko National University of Kyiv \\ Kyiv, Ukraine
}

Received 10.08.2021, received in revised form 20.09.2021, accepted 30.10.2021

\begin{abstract}
A method for the adsorption separation of inorganic arsenic species (As(III)/As(V)) using sequentially connected preconcentrating columns filled with functionalized silica gels and their determination by inductively coupled plasma optical emission spectrometry was proposed. As(V) was effectively retained at $\mathrm{pH} 3.5-6.5$ by an adsorbent containing groups of quaternary phosphonium bases on the surface and exhibiting the properties of an anion exchanger. In this $\mathrm{pH}$ range, As(III) was not extracted, which made it possible to separate As(V) from As(III). As(III) was retained in a wide pH range of 1-6 by a complexing adsorbent containing mercapto groups on the surface. Adsorbed As(V) was quantitatively eluted from the surface with $1 \mathrm{M} \mathrm{HNO}_{3}$, and As(III) - with $5 \%$ unithiol solution in $2 \mathrm{M}$ $\mathrm{HCl}$. The use of «non-aggressive» eluents allows us to reuse adsorbents for preconcentration of As(III) and $\mathrm{As}(\mathrm{V})$ at least 5 times. The separation efficiency was confirmed by the analysis of model solutions.
\end{abstract}

Keywords: arsenic, separation of chemical species, silica gel, adsorption preconcentration.

Acknowledgments. The research was funded by RFBR, Krasnoyarsk Territory and Krasnoyarsk Regional Fund of Science, project number 20-43-240006.

Citation: Borodina, E. V., Didukh-Shadrina, S. L., Losev, V.N., Trofimchuk, A. K. Adsorption separation of arsenic(III) and arsenic(V) using functionalized silica gels, J. Sib. Fed. Univ. Chem., 2021, 14(4), 477-488. DOI: 10.17516/1998-2836-0256

(C) Siberian Federal University. All rights reserved

This work is licensed under a Creative Commons Attribution-NonCommercial 4.0 International License (CC BY-NC 4.0).

* Corresponding author E-mail address: semdid@mail.ru 


\title{
Сорбционное разделение мышьяка(III) \\ и мышьяка(V) с помощью \\ функционализированных силикагелей
}

\author{
Е. В. Бородина ${ }^{a}$ С.Л. Дидух-Шадрина ${ }^{a}$, \\ В.Н. Лосев ${ }^{\mathrm{a}}$, А.К. Трофимчук ${ }^{0}$ \\ ${ }^{a}$ Научно-исследовательский инженерный центр «Кристалл» \\ Сибирского федерального университета \\ Российская Федерация, Красноярск \\ ${ }^{\sigma}$ Киевский нацииональный университет им. Тараса Шевченко \\ Украина, Киев
}

\begin{abstract}
Аннотация. Предложен способ сорбционного разделения неорганических форм мышьяка $(\mathrm{As}(\mathrm{III}) / \mathrm{As}(\mathrm{V}))$ с помощью функционализированных силикагелей с последующим определением методом атомно-эмиссионной спектроскопии с индуктивно связанной плазмой. $\mathrm{As}(\mathrm{V})$ эффективно удерживается при $\mathrm{pH}$ 3,5-6,5 сорбентом, содержащим на поверхности группы четвертичных фосфониевых оснований. В данном диапазоне $\mathrm{pH}$ отсутствует сорбция Аs(III), что позволяет отделять $\mathrm{As}(\mathrm{V})$ от $\mathrm{As}(\mathrm{III})$. Для извлечения $\mathrm{As}(\mathrm{III})$ предложен сорбент, функционализированный меркаптогруппами, который количественно извлекает As(III) в диапазоне pH 1-6. Сорбированный $\mathrm{As}(\mathrm{V})$ количественно элюируется с поверхности $1 \mathrm{M} \mathrm{HNO}_{3}$, a As(III) - раствором 5 \%-го унитиола в $2 \mathrm{M} \mathrm{HCl}$. Использование «неагрессивных» элюентов позволяет использовать сорбенты для повторного концентрирования $\mathrm{As}(\mathrm{III})$ и $\mathrm{As}(\mathrm{V})$ не менее 5 раз. Коэффициент концентрирования $\mathrm{As}(\mathrm{III})$ и $\mathrm{As}(\mathrm{V})$ равен 20. Эффективность разделения подтверждена анализом модельных растворов.
\end{abstract}

Ключевые слова: мышьяк, разделение химических форм, силикагель, сорбционное концентрирование.

Благодарность. Исследование выполнено при финансовой поддержке РФФИ, Правительства Красноярского края и Красноярского краевого фонда науки в рамках научного проекта № 2043-240006.

Цитирование: Бородина, Е.В. Сорбционное разделение мышьяка(II) и мышьяка(V) с помощью функционализированных силикагелей / Е. В. Бородина, С. Л. Дидух-Шадрина, В. Н. Лосев, А. К. Трофимчук // Журн. Сиб. федер. ун-та. Химия, 2021, 14(4). C. 477-488. DOI: 10.17516/1998-2836-0256

Мышьяк широко распространен в атмосфере, почвах, природных водах и живых организмах из-за интенсивного использования мышьяксодержащих продуктов (пестицидов, гербицидов, консервантов), из-за вулканической деятельности, сжигания реактивного топлива и мусора. Последнее время большое внимание уделяется загрязнению мышьяком, так как это один из токсичных элементов, который может накапливаться по пищевой цепочке и представлять угрозу для здоровья людей [1]. Также мышьяк может попадать из подземных вод в почвы, а из них в растения и, в конечном счете, в организм человека. Высокое содержание мышьяка 
в объектах окружающей среды провоцирует различные заболевания, в том числе некоторые виды рака [2].

В природе мышьяк подвергается биохимическому воздействию, претерпевая окислительновосстановительные превращения, поэтому данный элемент присутствует в окружающей среде в виде нескольких форм, которые обладают различной подвижностью, биодоступностью и токсичностью [3]. В целом, неорганические формы мышьяка более токсичны, чем органические, а токсичность As(III) в 10-20 раз выше, чем As(V) [4]. Это связано с тем, что As(III) взаимодействует с тиольными группами цистеина и метионина в составе белков-ферментов, блокируя их работу. Арсенат в почвах и донных отложениях может также восстанавливаться до арсенита в результате жизнедеятельности бактерий [5].

Дополнительную трудность при анализе создает взаимный переход форм мышьяка друг в друга при обычных атмосферных условиях, поскольку в отобранных образцах воды при хранении протекают окислительно-восстановительные процессы, которые искажают результаты определений. Для решения этой проблемы авторы работы [6] проводили разделение мышьяка(III) и мышьяка(V) непосредственно сразу после отбора проб в полевых условиях.

Аналитические методы для разделения и концентрирования форм мышьяка рассмотрены в ряде обзоров [7-10]. Основным подходом в разделении и концентрировании неорганических форм мышьяка служит сочетание эффективного метода разделения с чувствительным методом определения. В целом, методы определения форм мышьяка можно разделить на хроматографические и нехроматографические. Несмотря на то, что хроматографические методы обладают рядом непревзойденных достоинств, нехроматографические методы отличаются значительно большей чувствительностью, простотой, экономичностью и доступностью, поэтому их развитие является актуальной задачей. Особо при этом стоит выделить сорбционный метод, который обеспечивает высокую эффективность разделения форм, высокие коэффициенты концентрирования, простоту, экспрессность и экономичность.

В качестве сорбентов для разделения и конценрирования форм мышьяка используется множество материалов различной природы: разнообразные смолы [11-15], активные угли [16], углеродные нанотрубки [17, 18], магнитные сорбенты [19], биосорбены [20] и т. п., однако наиболее широко применяются материалы на основе оксида кремния [21-23], в частности силикагель. Силикагели с закрепленными функциональными группами различной природы хорошо изучены и находят повсеместное применение, так как обладают рядом ценных свойств, а полученные с их применением результаты легко воспроизводятся.

Сорбенты, селективно извлекающие $\mathrm{As}(\mathrm{V})$, содержат, как правило, анионообменные группы, ибо мышьяковая кислота представлена в водных растворах преимущественно анионной формой, а сорбенты, селективно извлекающие As(III), включают серусодержащие комплексообразующие группы, ибо диссоциация мышьяковой кислоты начинается только при $\mathrm{pH}>9$. Смешанные сорбенты, содержащие и ионообменные, и комплексообразующие группы, например кремнезем, химически модифицированный меркапто- и аминогруппами, способны извлекать обе формы мышьяка одновременно [23].

Для последующего определения мышьяка атомно-спектральными методами анализа необходимо проведение стадии элюирования. Особую сложность при этом представляет элюирование $\mathrm{As}(\mathrm{III})$ с поверхности серосодержащих сорбентов, на которой он очень прочно удержи-

$$
-479-
$$


вается. Большинство исследователей предлагают в качестве элюента использовать растворы $\mathrm{NaOH}$ различной концентрации [24], которые фактически частично разрушают верхний слой матрицы кремнезема и смывают As вместе с функциональными группами сорбента. В этом случае сорбент не регенерируем, а элюат представляет собой щелочной раствор, содержащий кремниевую кислоту, которая повреждает кварцевую распылительную систему ИСП-АЭС и ИСП-МС спектрометров.

Поэтому сорбционное разделение $\mathrm{As}(\mathrm{V})$ и $\mathrm{As}(\mathrm{III})$ с использованием регенерируемых сорбентов и последующее их определение является актуальной задачей.

В данной работе для сорбционного разделения и концентрирования $\mathrm{As}(\mathrm{V})$ и $\mathrm{As}(\mathrm{III})$ перед их последующим определением атомно-эмиссионным с индуктивно связанной плазмой методом использована двухколоночная система, содержащая два типа функционализированных силикагелей - с трифенилфосфониевыми группами и меркаптопропильными группами.

\section{Экспериментальная часть}

Приборы. Распределение мышьяка между водной фазой и сорбентом контролировали по анализу водной фазы методом ИСП-АЭС с использованием спектрометра iCAP 6500 Duo (Thermo Scientific, США). Оптимальные параметры работы прибора представлены в табл. 1. pH растворов измеряли с помощью иономера SevenMulti (Mettler-Toledo, Швейцария) с комбинированным электродом InLabExpertPro. Перистальтический насос Masterflex L/S (ColeParmer Instruments Company, США) применяли для прокачивания жидкостей через стеклянные миниколонки (внутренний диаметр 3 мм, высота 5 см), заполненные сорбентом. Концентрацию трифенилфосфониевых и меркаптопропильных групп, закрепленных на поверхности кремнезема, определяли методом элементного анализа на анализаторе HCNS-0 EA 1112 (Flash, США).

Реактивы. Исходные растворы As(III) с концентрацией 5 мг/л готовили из стандартного раствора ионов ГСО № 7976-2001 растворением аликвоты в дистиллированной воде. Исходные растворы $\mathrm{As}(\mathrm{V})$ с концентрацией 5 мг/л готовили окислением стандартного раствора ионов ГCO As(III) № 7976-2001 кипячением с пероксидом водорода. Полученный раствор доводили до объема 50 мл. Необходимое значение $\mathrm{pH}$ создавали растворами $\mathrm{HCl}, \mathrm{NaOH}$ квали-

Таблица 1. Параметры ИСП-АЭС определения

Table 1. ICP-OES operational conditions

\begin{tabular}{ll}
\hline \multicolumn{1}{c}{ Характеристики } & Параметры \\
\hline Мощность генератора плазмы & 1150 Вт \\
Распылительный поток & 0.7 л/мин \\
Охлаждающий поток & 12 л/мин \\
Вспомогательный поток & 0.5 л/мин \\
Скорость насоса & 60 об/мин \\
Время стабилизации насоса & 5 с \\
Время интегрирования сигнала & 15 с \\
Обзор плазмы & аксиальный \\
Количество измерений & 3 \\
\hline
\end{tabular}


Таблица 2. Реагенты для элюирования As(III)

Table 2. Reagents for As(III) elution

\begin{tabular}{|c|c|c|}
\hline $\begin{array}{c}\text { Полное название реагента } \\
\text { (Сокращенное название) }\end{array}$ & Структурная формула & $\begin{array}{l}\text { Производитель, } \\
\text { CAS № }\end{array}$ \\
\hline 3-меркапто-1-пропан сульфонат натрия (МП) & $\mathrm{Na}$ & $\begin{array}{l}\text { Sigma-Aldrich, } \\
17636-10-1\end{array}$ \\
\hline 2-меркаптоэтансульфонат натрия (МЭС) & $\mathrm{HS}^{-}$ & $\begin{array}{l}\text { Sigma-Aldrich, } \\
19767-45-4\end{array}$ \\
\hline $\begin{array}{l}\text { 2-меркапто-5-бензимидазол сульфокислота } \\
\text { натривая соль (МБИ) }\end{array}$ & & $\begin{array}{l}\text { Sigma-Aldrich, } \\
\text { 207511-11-3 }\end{array}$ \\
\hline Тиомочевина & & ООО «Универхимик» \\
\hline 2,3-дитиол-1-пропансульфонат натрия (унитиол) & HS & $\begin{array}{l}\text { Sigma-Aldrich, } \\
4076-02-2\end{array}$ \\
\hline
\end{tabular}

фикации х. ч. Реактивы, используемые в качестве десорбирующих растворов (табл. 2), имели квалификацию х. ч. В качестве основы для синтеза сорбента взяли кремнезем марки Силохром С-80 (Люминофор, Россия), фракция 0,1-0,2 мм, удельная поверхность 80 м²/г, средний диаметр пор 50 нм.

Синтез сорбентов. Синтезы функционализированного кремнезема меркаптопропильными (МПС) или трифенилфосфониевыми группами (ТФФС) проводили по методикам $[25,26]$.

Методика эксперимента. Зависимость степени извлечения $\mathrm{As}(\mathrm{III})$ и $\mathrm{As}(\mathrm{V})$ от $\mathrm{pH}$ и времени контакта фаз исследовали в статическом режиме. Для этого в стеклянную пробирку с притертой пробкой вводили раствор As(III) или $\mathrm{As}(\mathrm{V})$, содержащий 10 мкг As, добавляли $\mathrm{HCl}$ или $\mathrm{NaOH}$ для создания необходимого значения $\mathrm{pH}$, воду до общего объема 10 мл, вносили 0,1 г сорбента ТФФС или МПС и встряхивали в течение 5-30 мин в зависимости от целей эксперимента. Раствор отделяли от сорбента декантацией и определяли в нем остаточное содержание мышьяка методом ИСП-АЭС при длине волны 189,042 нм.

При исследовании сорбции в динамическом режиме в стеклянную миниколонку помещали 0,1 г сорбента ТФФС или МПС и при помощи перистальтического насоса пропускали через нее раствор $\mathrm{As}(\mathrm{V})$ или $\mathrm{As}(\mathrm{III})$ соответственно. Остаточное содержание мышьяка определяли в каждых 10 мл прошедшего раствора методом ИСП-АЭС. По полученным результатам строили динамические выходные кривые.

Для десорбции мышьяка в динамическом режиме через стеклянную миниколонку, содержащую 0,1 г сорбента ТФФС или МПС с сорбированным $\mathrm{As}(\mathrm{V})$ или $\mathrm{As}(\mathrm{III})$ соответственно в количестве 10 мкг, пропускали 5 и 10 мл десорбирующего раствора со скоростью потока 1 мл/мин. Содержание мышьяка в растворе после десорбции определяли методом ИСП-АЭС.

$$
-481-
$$




\section{Результаты и обсуждение}

Сорбиионное извлечение $A s(I I I)$ u $A s(V)$. Количество трифенилфосфониевых и меркаптопропильных групп, закрепленных на поверхности кремнеземов, по данным элементного анализа, составило 0,08 и 0,6 ммоль/г соответственно. Функционализированную поверхность синтезированных сорбентов можно представить в следующем виде:

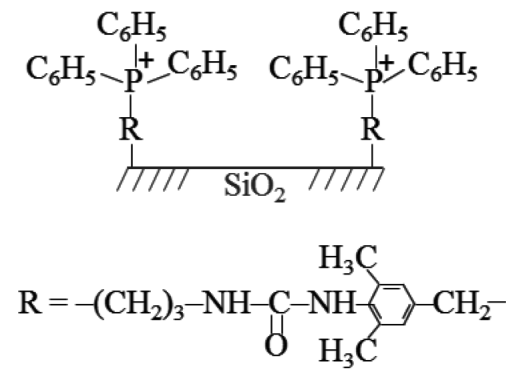

ТФФС

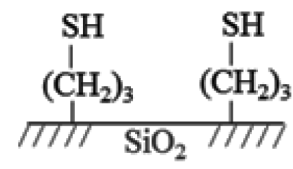

MIIC

Важным фактором, определяющим распределение и разделение видов мышьяка, является pH раствора. ТФФС, имеющий положительно заряженную поверхность и выступающий как типичный анионообменник, извлекает $\mathrm{As}(\mathrm{V})$ из водных растворов при $\mathrm{pH}>2,0$ (рис. 1, кривая 1). Максимальная степень извлечения достигается при $\mathrm{pH} 3,5-6,5$ и составляет $\geq 95 \%$. При данных значениях $\mathrm{pH} \mathrm{As}(\mathrm{V})$ существует в водных растворах в виде арсенат-ионов: $\mathrm{H}_{2} \mathrm{AsO}_{4}$ $\left(\mathrm{pK}_{\mathrm{a} 1}=2,3\right), \mathrm{HAsO}_{4}{ }^{2-}\left(\mathrm{pK}_{\mathrm{a} 2}=7,0\right)$ [24]. Взаимодействие между $\mathrm{As}(\mathrm{V})$ и трифенилфосфониевыми группами при $\mathrm{pH}<3$ значительно снижается, что связано с существованием $\mathrm{As}(\mathrm{V})$ в нейтральной молекулярной форме. 50\%-ная сорбция $\mathrm{As}(\mathrm{V})$ наблюдается при $\mathrm{pH}=2,5$, что близко к значению рКа 1 ионизации мышьяковой кислоты по первой ступени. Время установления сорбционного равновесия при рН 3,5-6,5 не превышает 10 мин.

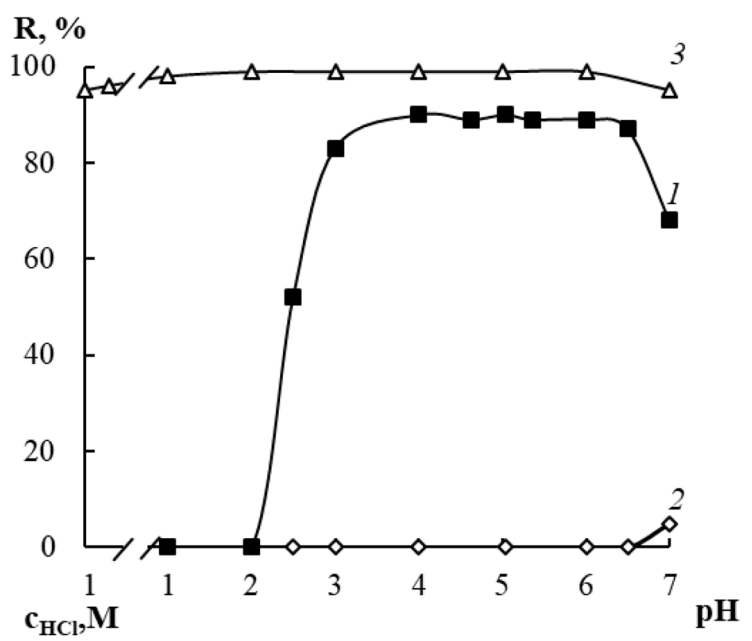

Рис. 1. Степень извлечения $\mathrm{As}(\mathrm{V})(1)$ и $\mathrm{As}(\mathrm{III})(2,3)$ сорбентами ТФФС $(1,2)$ и МПС (3) от рН раствора: $\mathrm{m}_{\text {сорбента }}=0,1$ г, $\mathrm{C}_{(\mathrm{As})}=1$ мкг $/ \mathrm{мл}, \mathrm{V}_{\mathrm{p} \text {-ра }}=10$ мл

Fig. 1. Extraction of $\operatorname{As}(\mathrm{V})(1)$ and $\operatorname{As}(\mathrm{III})(2,3)$ by TPPS $(1,2)$ and MPS (3) sorbents vs. $\mathrm{pH}: \mathrm{m}_{\text {adsorbent }}=0.1 \mathrm{~g}$, $\mathrm{C}_{(\mathrm{As})}=1 \mu \mathrm{g} \mathrm{mL}^{-1}, \mathrm{~V}_{\mathrm{sol}}=10 \mathrm{~mL}$ 
Мышьяк(III) находится в растворе при данных условиях в виде недиссоциированной мышьяковистой кислоты ( $\left.\mathrm{pK}_{\mathrm{al}}=9.2\right)$ и не извлекается сорбентом ТФФС (рис. 1, кривая 2).

Высокую сорбционную эффективность по отношению к Аs(III) проявляет сорбент МПС. Количественное извлечение (степень извлечения $\mathrm{R} \geq 98$ \%) $\mathrm{As}(\mathrm{III})$ достигается в диапазоне $\mathrm{pH}$ 1,0-6,0. В 0,5-1 M HCl степень извлечения снижается до $95 \%$ (рис. 1, кривая 3), что может быть связано с частичным окислением As(III) до As (V). Таким образом, при использовании сорбентов ТФФС и МПС можно осуществить разделение и концентрирование $\mathrm{As}(\mathrm{V})$ и $\mathrm{As}(\mathrm{III})$.

Применение динамического концентрирования позволяет достигать количественного извлечения (степень извлечения $\geq 98$ \%) As(V) сорбентом ТФФС в диапазоне $\mathrm{pH}$ 3,5-7,0 из больших объемов растворов. Максимальный объем раствора $\mathrm{As}(\mathrm{V})$ с концентрацией 1 мкг/мл, из которого наблюдается его количественное извлечение, составляет 70 мл при $\mathrm{pH}=5$ (рис. 2, кривая 1) при скорости потока раствора 1 мл/мин. С уменьшением исходной концентрации $\mathrm{As}(\mathrm{V})$ в растворе в 2 раза до 0,5 мкг/мл пропорционально увеличивается объем раствора, пропускаемого через колонку, и составляет 140 мл до точки проскока (рис. 2, кривая 2). Динамическая сорбционная емкость сорбента ТФФС, рассчитанная до точки проскока, равна 9,3 мкмоль $\mathrm{As}(\mathrm{V})$ на 1 г, что соответствует 700 мкг $\mathrm{As}(\mathrm{V})$ на 1 г сорбента.

В динамическом режиме при исходной концентрации As(III) 1 мкг/мл его извлечение наблюдается во всем пропускаемом объеме раствора - 240 мл (рис. 2, кривая 3). Заметный выход мышьяка (5 \%) наблюдается при прохождении 200 мл. Однако при дальнейшем увеличении объема степень его извлечения не изменяется. Сорбционная емкость сорбента МПС до точки проскока составляет 30 мкмоль As(III) на 1 г сорбента.

Влияние сопутствующих ионов на сорбиионное извлечение $A s(I I I)$ и As(V). На сорбционное концентрирование анионных форм $\mathrm{As}(\mathrm{V})$ будут оказывать влияние различные отрицательно заряженные ионы, присутствующие в природных водах и извлекающиеся положительно заряженной поверхностью кремнезема с четвертичными фосфониевыми основаниями. Исследова-

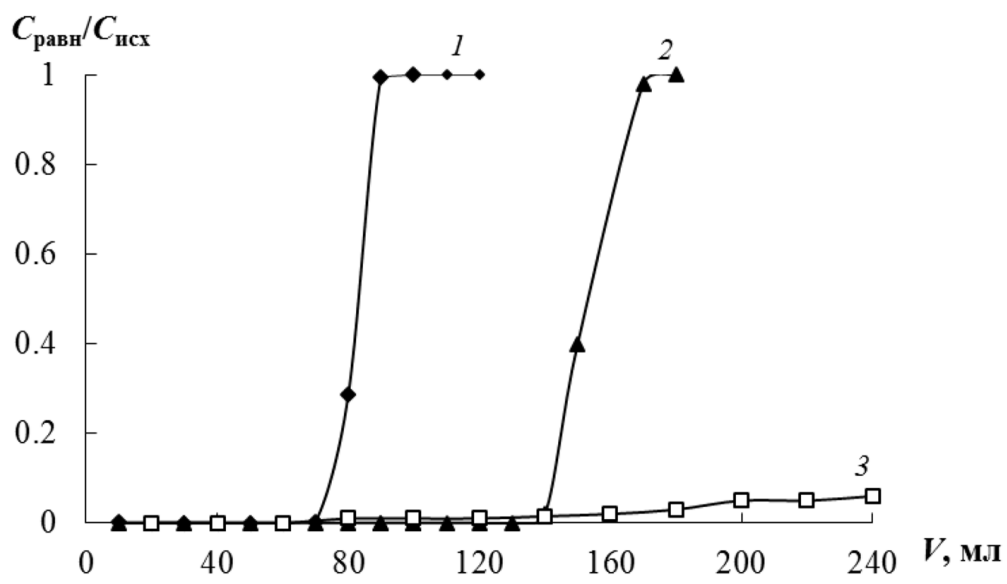

Рис. 2. Динамические выходные кривые $\mathrm{As}(\mathrm{V})(1,2)$ и $\mathrm{As}(\mathrm{III})(3): \mathrm{m}_{\text {сорбента }}=0,1$ г, $\mathrm{C}_{(\mathrm{As})}=1$ мкг/мл $(1,3)$, 0,5 мкг/мл (2), скорость потока 1 мл/мин, $\mathrm{pH}=5$

Fig. 2. Breakthrough curves for $\mathrm{As}(\mathrm{V})(1,2)$ and $\mathrm{As}(\mathrm{III})(3)$ : $\mathrm{m}_{\text {adsorbent }}=0.1 \mathrm{r}, \mathrm{C}_{(\mathrm{As})}=1 \mu \mathrm{g} \mathrm{mL} \mathrm{L}^{-1}(1,3), 0,5 \mu \mathrm{g} \mathrm{mL}-1$ (2), flow rate $1 \mathrm{ml} \mathrm{min}^{-1}, \mathrm{pH}=5$ 
ние влияния анионов - хлоридов, гидрокарбонатов, нитратов и сульфатов - проводили в динамическом режиме в бинарных растворах, содержащих 1 мкг/мл $\mathrm{As}(\mathrm{V})$ и 5-50 мг/л стороннего аниона при скорости потока раствора 1 мл/мин.

На извлечение $\mathrm{As}(\mathrm{V})$ сорбентом ТФФС не влияют однозарядные анионы с концентрацией 5 и 10 мг/л. Присутствие хлорид-иона с концентрацией 40 мг/л и сульфат-иона с концентрацией 5 мг/л снижает динамическую емкость сорбента по отношению к $\mathrm{As}(\mathrm{V})$ до точки проскока в три раза до 3 мкмоль/г.

На сорбционное извлечение As(III) сорбентом МПС могут оказывать влияние цветные и тяжелые металлы, образующие комплексные соединения с SH-группами сорбента. Поэтому для определения возможности реализации последовательного разделения и концентрирования $\mathrm{As}(\mathrm{III})$ и $\mathrm{As}(\mathrm{V})$ изучали селективность сорбции $\mathrm{As}(\mathrm{III})$ из 100 мл раствора с его концентрацией 1 и 0.1 мкг/мл сорбентом МПС при $\mathrm{pH} 2$, при котором наблюдается максимальное отличие его степени извлечения $\mathrm{As}(\mathrm{III})$ от $\mathrm{As}(\mathrm{V})$, и при $\mathrm{pH}$ 5, при котором наблюдается количественное извлечение как As(III) сорбентом MПC, так и As(V) сорбентом ТФФС (рис. 1).

Мешающее влияние сторонних катионов считалось значительным, если степень извлечения As(III) изменялась более чем на $\pm 5 \%$. Количественному извлечению 1 мкг/мл As(III) при pH 2 не мешают (в кратных количествах): $\mathrm{Na}^{+}, \mathrm{K}^{+}, \mathrm{Ca}^{2+}, \mathrm{Mg}^{2+}$ (1000), $\mathrm{Zn}^{2+}, \mathrm{Al}^{3+}, \mathrm{Fe}^{3+}$ (100), $\mathrm{Ni}^{2+}$, $\mathrm{Pb}^{2+}, \mathrm{Cd}^{2+}(50)$. Катионы $\mathrm{Cu}^{2+}$ извлекаются в том же диапазоне $\mathrm{pH}$, что и $\mathrm{As}(\mathrm{III})$, однако не снижают его степень извлечения из 100 мл раствора при концентрации 1 мкг/мл. При уменьшении концентрации $\mathrm{As}(\mathrm{III})$ в растворе до 0,1 мкг/мл селективность его извлечения увеличивается в присутствии $\mathrm{Zn}^{2+}, \mathrm{Al}^{3+}, \mathrm{Fe}^{3+}, \mathrm{Ni}^{2+}, \mathrm{Pb}^{2+}, \mathrm{Cd}^{2+}, \mathrm{Cu}^{2+}$ в 10 раз. Высокая селективность извлечения $\mathrm{As}(\mathrm{III})$ связана с тем, что при $\mathrm{pH} 2$ данные элементы не извлекаются сорбентом МПС.

При рН 5 сорбент МПС проявляет меньшую селективность, поскольку при данном значении $\mathrm{pH}$ наблюдается извлечение $\mathrm{Zn}^{2+}, \mathrm{Fe}^{3+}, \mathrm{Ni}^{2+}, \mathrm{Pb}^{2+}, \mathrm{Cd}^{2+}, \mathrm{Cu}^{2+}$. Сорбционному извлечению $\mathrm{As}(\mathrm{III})$ из 100 мл раствора с его концентрацией 1 мкг/мл не мешают 10-кратные избытки данных катионов металлов. При уменьшении концентрации $\mathrm{As}(\mathrm{III})$ до 0.1 мкг/мл селективность извлечения As(III) сорбентом МПС увеличивается в 10 раз. Таким образом, при уменьшении концентрации $\mathrm{As}(\mathrm{III})$ в растворе увеличивается селективность его извлечения.

Высокая селективность извлечения As(III) при рH 5 позволяет осуществлять его выделение из природных вод сорбентом МПС на фоне сопутствующих ионов металлов и реализовать последовательное выделение и концентрирование $\mathrm{As}(\mathrm{V})$ сорбентом ТФФС и $\mathrm{As}(\mathrm{III})$ сорбентом МПС из водных растворов при одном значении $\mathrm{pH}=5$. Таким образом, предлагаемые сорбенты являются селективным для выделения $\mathrm{As}(\mathrm{III})$ и $\mathrm{As}(\mathrm{V})$ и могут быть применены для их концентрирования и определения в реальных объектах.

Десорбция $A s(I I I)$ и $A s(V)$. Десорбцию $\mathrm{As}(\mathrm{III})$ и $\mathrm{As}(\mathrm{V})$ изучали в динамическом режиме при скорости потока 1 мл/мин.

Поскольку извлечение $\mathrm{As}(\mathrm{V})$ сорбентом ТФФС происходит за счет анионообменного механизма и при $\mathrm{pH} 1 \mathrm{As}(\mathrm{V})$ не извлекается, то в качестве десорбирующих были выбраны растворы минеральных кислот. Количественная десорбция $\left(\mathrm{R}_{\text {десорб }}>98\right.$ \%) мышьяка с поверхности ТФФС достигается 5 и 10 мл $1 \mathrm{M} \mathrm{HNO}_{3}$ или $\mathrm{HCl}$.

Особую сложность представляет десорбция Аs(III) с поверхности МПС, образующего прочные комплексы с меркаптогруппами. Выбор серосодержащих реагентов для десорбции 
As(III) (табл. 2) основывался на том, что они также образуют прочные комплексы с As(III) и их концентрация в растворе значительно превышает концентрацию привитых меркаптопропильных групп. Степень десорбции As(III) с поверхности сорбента МПС с использованием различных реагентов и их концентрации приведена в табл. 3. Как видно по данным этой таблицы, максимальная степень десорбции As(III) (99 \%) достигается при использовании 5 мл 5 \%-ного раствора унитиола в $2 \mathrm{M} \mathrm{HCl}$.

При использовании данных элюирующих растворов сорбционные свойства ТФФС и МПС по отношению к $\mathrm{As}(\mathrm{V})$ и $\mathrm{As}(\mathrm{III})$ соответственно не изменяются как минимум после пяти циклов сорбции-десорбции.

Достигаемый коэффициент концентрирования при сорбции 0,5 мкг/мл мышьяка из 100 мл раствора и объемом элюента 5 мл равен 20 для $\mathrm{As}(\mathrm{V})$ и $\mathrm{As}(\mathrm{III})$.

Методика разделения $A s(I I I)$ u $A s(V)$. На основании полученных результатов сорбционного концентрирования $\mathrm{As}(\mathrm{V})$ и $\mathrm{As}(\mathrm{III})$ с использованием сорбентов ТФФС и МПС предложен двухколоночный вариант разделения [27].

Раствор, содержащий $\mathrm{As}(\mathrm{V})$ и $\mathrm{As}(\mathrm{III})$, объемом 100 мл при $\mathrm{pH}=5$ пропускают через две последовательно соединенные миниколонки со скоростью потока раствора 1 мл/мин. В первой колонке, содержащей 0,1 г сорбента ТФФС, происходит сорбционное извлечение $\mathrm{As}(\mathrm{V})$, во второй,

Таблица 3. Десорбция As(III) с поверхности МПС

Table 3. Desorption of As(III) from MPS surface

\begin{tabular}{cccccc}
\hline \multirow{2}{*}{ Реагент } & $\begin{array}{c}\text { Концентрация / } \\
\text { Среда }\end{array}$ & $\mathrm{R}_{\text {десорб} \%} \%$ & Реагент & $\begin{array}{c}\text { Концентрация / } \\
\text { Среда }\end{array}$ & $\mathrm{R}_{\text {десорб, }} \%$ \\
\hline \multirow{2}{*}{ МП } & $1 \% / 2 \mathrm{M} \mathrm{HCl}$ & 2 & Унитиол & $0.1 \% / \mathrm{H}_{2} \mathrm{O}$ & 2 \\
& $5 \% / 2 \mathrm{M} \mathrm{HCl}$ & 49 & & $0.5 \% / \mathrm{H}_{2} \mathrm{O}$ & 7 \\
\multirow{2}{*}{ МЭС } & $1 \% / 2 \mathrm{M} \mathrm{HCl}$ & 2 & & $1 \% / \mathrm{H}_{2} \mathrm{O}$ & 12 \\
МБИ & $1 \% / 2 \mathrm{M} \mathrm{HCl}$ & 0 & $2.5 \% / \mathrm{H}_{2} \mathrm{O}$ & 24 \\
\multirow{2}{*}{ Тиомочевина } & $10 \% / \mathrm{H}_{2} \mathrm{O}$ & 0 & $5 \% / \mathrm{H}_{2} \mathrm{O}$ & 27 \\
& $10 \% / 2 \mathrm{M} \mathrm{HCl}$ & 1 & & $0.5 \% / 2 \mathrm{M} \mathrm{HCl}$ & 58 \\
& $10 \% / \mathrm{H}_{2} \mathrm{O}$ & 2 & & $5 \% / 2 \mathrm{M} \mathrm{HCl}$ & 99 \\
\hline
\end{tabular}

Таблица 4. Результаты разделения и определения $\mathrm{As}(\mathrm{V})$ и $\mathrm{As}(\mathrm{III})$ в модельных растворах $(\mathrm{n}=3, \mathrm{P}=0,95)$

Table 4. Results of separation and determination of $\mathrm{As}(\mathrm{V})$ and $\mathrm{As}(\mathrm{III})$ in model solutions $(\mathrm{n}=3, \mathrm{P}=0,95)$

\begin{tabular}{cccc}
\hline \multicolumn{2}{c}{ Концентрация мышьяка в растворе, мкг/л } & \multicolumn{2}{c}{ Найдено мышьяка, мкг/л } \\
\hline $\operatorname{As}(\mathrm{V})$ & $\operatorname{As}(\mathrm{III})$ & $\operatorname{As}(\mathrm{V})$ & $\operatorname{As}(\mathrm{III})$ \\
\hline 10 & 20 & $10.6 \pm 0.7$ & $20.9 \pm 1.1$ \\
20 & 80 & $20.8 \pm 1.0$ & $79.4 \pm 2.7$ \\
80 & 80 & $81.3 \pm 2.6$ & $78.5 \pm 2.6$ \\
20 & 10 & $20.7 \pm 1.0$ & $9.9 \pm 0.7$ \\
80 & 10 & $80.2 \pm 1.8$ & $9.8 \pm 0.7$ \\
\hline
\end{tabular}


содержащей 0,1 г сорбента MПC, - As(III). Десорбцию As(V) и As(III) осуществляют раздельным пропусканием через первую колонку 5 мл $1 \mathrm{M} \mathrm{HNO}_{3}$, через вторую - 5 мл 5 \%-ного унитиола в 2 M HCl. Содержание мышьяка в десорбирующих растворах определяют методом ИСП-АЭС.

Эффективность разделения и последующего определения форм мышьяка при их совместном присутствии подтверждена анализом модельных растворов, содержащих различные концентрации $\mathrm{As}(\mathrm{V})$ и $\mathrm{As}(\mathrm{III})$ (табл. 4).

\section{Заключение}

Предложена методика концентрирования и разделения $\mathrm{As}(\mathrm{III})$ и $\mathrm{As}(\mathrm{V})$ с использованием двух последовательно соединенных концентрирующих колонок, содержащих кремнезем, функционализированный трифенилфосфониевыми и меркаптопропильными группами соответственно. В первой колонке происходит селективное концентрирование $\mathrm{As}(\mathrm{V})$ за счет электростатического взаимодействия с положительно заряженными трифенилфосфониевыми группами, а во второй - As(III) за счет комплексообразования с меркаптопропильными группами. Коэффициенты концентрирования составляют 20 для As(V) и As(III).

\section{Список литературы / References}

1. Smedley, P. L., Kinniburgh, D. G. A review of the source, behaviour and distribution of arsenic in natural waters. Applied Geochemistry 2002. Vol. 17(5), P. 517-568. doi:10.1016/S08832927(02)00018-5

2. Karagas, M. R., Stukel, T. A., Tosteson, T.D. Assessment of cancer risk and environmental levels of arsenic in new hampshire. International Journal of Hygieneand Environmental Health 2002. Vol. 205(1-2), P. 85-94. doi:10.1078/1438-4639-0013

3. Tamaki, S., Frankenberger Jr., W.T. Environmental biochemistry of arsenic. Reviews of Environmental Contamination and Toxicology 1992. Vol. 124, P. 79-110.

4. Qingxiang Zhou, Zhenwen Zheng, Junping Xiao, Huili Fan. Sensitive determination of As (III) and As (V) by magnetic solid phase extraction with Fe@polyethyleneimine in combination with hydride generation atomic fluorescence spectrometry. Talanta 2016. Vol. 156-157, P. 196-203. doi: 10.1016/j.talanta.2016.05.024.

5. Al Lawati, W. M., Rizoulis, A., Eiche, E., Boothman, C., Polya, D. A., Lloyd, J. R., Van Dongen, B.E. Characterisation of organic matter and microbial communities in contrasting arsenicrich holocene and arsenic-poor pleistocene aquifers, red river delta, vietnam. Applied Geochemistry 2012. Vol. 27(1), P. 315-325. doi:10.1016/j.apgeochem.2011.09.030

6. Kim, M., Nriagu, J., \& Haack, S. Arsenic species and chemistry in groundwater of southeast michigan. Environmental Pollution 2002. Vol. 120(2), 379-390. doi:10.1016/S0269-7491(02)00114-8

7. Katarzyna Wrobel, Kazimierz Wrobel, Joseph A Caruso. Pretreatment procedures for characterization of arsenic and selenium species in complex samples utilizing coupled techniques with mass spectrometric detection. Analytical and bioanalytical chemistry 2002. Vol. 381(2), P. 317-331.doi: 10.1007/s00216-004-2959-5

8. Braeuer, S., Goessler, W. Arsenic species in mushrooms, with a focus on analytical methods for their determination - A critical review. Analytica Chimica Acta 2019. Vol. 1073, P. 1-21. doi:10.1016/j. aca.2019.04.004 
9. Yu, X., Liu, C., Guo, Y., Deng, T. Speciation analysis of trace arsenic, mercury, selenium and antimony in environmental and biological samples based on hyphenated techniques. Molecules 2019, doi:10.3390/molecules24050926

10. Xu, F., Hu, J., Zhang, J., Hou, X., Jiang, X. Nanomaterials in speciation analysis of mercury, arsenic, selenium, and chromium by analytical atomic/molecular spectrometry. Applied Spectroscopy Reviews 2018. Vol. 53(2-4), P. 333-348. doi:10.1080/05704928.2017.1323310

11. Issa, N. B., Rajaković-Ognjanović, V. N., Marinković, A. D., Rajaković, L. V. Separation and determination of arsenic species in water by selective exchange and hybrid resins. Analytica Chimica Acta 2011. Vol. 706(1), P. 191-198. doi:10.1016/j.aca.2011.08.015

12. Tunçeli, A., Ocak, G., Acar, O., Türker, A.R. Development of a method for speciation of inorganic arsenic in waters using solid phase extraction and electrothermal atomic absorption spectrometry. International Journal of Environmental Analytical Chemistry 2015. Vol. 95(14), P. 13951411. doi:10.1080/03067319.2015.1100728

13. Issa, N. B., Rajaković-Ognjanović, V. N., Jovanović, B. M., Rajaković, L. V. Determination of inorganic arsenic species in natural waters-benefits of separation and preconcentration on ion exchange and hybrid resins. Analytica Chimica Acta 2010. Vol. 673(2), P. 185-193. doi:10.1016/j.aca.2010.05.027

14. Barakat, M. A., Ismat-Shah, S. Utilization of anion exchange resin Spectra/Gel for separation of arsenic from water. Arabian Journal of Chemistry 2013. Vol. 6(3), P. 307-311. doi:10.1016/j. arabjc.2010.10.011

15. Tuzen, M., Saygi, K. O., Karaman, I., Soylak, M. Selective speciation and determination of inorganic arsenic in water, food and biological samples. Food and Chemical Toxicology 2010. Vol. 48(1), P. 41-46. doi:10.1016/j.fct.2009.09.012

16. Ryu, S., Jeon, E., Yang, J., Baek, K. Adsorption of As(III) and As(V) in groundwater by FeMn binary oxide-impregnated granular activated carbon (IMIGAC). Journal of the Taiwan Institute of Chemical Engineers 2017. Vol. 72, P. 62-69. doi:10.1016/j.jtice.2017.01.004

17. Wu, H., Wang, X., Liu, B., Liu, Y., Li, S., Lu, J., Yang, Z. Simultaneous speciation of inorganic arsenic and antimony in water samples by hydride generation-double channel atomic fluorescence spectrometry with on-line solid-phase extraction using single-walled carbon nanotubes microcolumn. Spectrochimica Acta - Part B Atomic Spectroscopy 2011. Vol. 66(1), P. 74-80. doi:10.1016/j. sab.2010.12.002

18. Letsoalo, M. R., Godeto, T.W., Magadzu, T., Ambushe, A. A. Selective speciation of inorganic arsenic in water using nanocomposite based solid-phase extraction followed by inductively coupled plasma-mass spectrometry detection, Journal of Environmental Science and Health, Part A 2019. Vol. 54(9), P. 924-932, doi: 10.1080/10934529.2019.1609321

19. Zhou, Q., Zheng, Z., Xiao, J., Fan, H. Sensitive determination of As (III) and As (V) by magnetic solid phase extraction with Fe@polyethyleneimine in combination with hydride generation atomic fluorescence spectrometry. Talanta 2016. Vol. 156-157, P. 196-203. doi:10.1016/j.talanta.2016.05.024

20. Santos, L. B., de Oliveira, D. M., de Souza, A. O., Lemos, V. A. A new method for the speciation of arsenic species in water, seafood and cigarette samples using an eggshell membrane. Journal of the Iranian Chemical Society 2019. Vol. 16(9), P. 1879-1889. doi:10.1007/s13738-019-01665-8

21. Xiong, C., He, M., Hu, B. On-line separation and preconcentration of inorganic arsenic and selenium species in natural water samples with CTAB-modified alkyl silica microcolumn and

$$
-487 \text { - }
$$


determination by inductively coupled plasma-optical emission spectrometry. Talanta 2008. Vol. 76(4), P. 772-779. doi:10.1016/j.talanta.2008.04.031

22. Terada, K., Matsumoto, K., Inaba, T. Differential preconcentration of arsenic(III) and arsenic(V) with thionalide loaded on silica gel. Analytica Chimica Acta 1984. Vol. 158, P. 207-215. doi:10.1016/s0003-2670(00)84828-x

23. Boyac, E., Çağır, A., Shahwan, T., Eroğlu, A. E. Synthesis, characterization and application of a novel mercapto- and amine-bifunctionalized silica for speciation/sorption of inorganic arsenic prior to inductively coupled plasma mass spectrometric determination. Talanta 2011. Vol. 85(3), P. 15171525. doi:10.1016/j.talanta.2011.06.021

24. Issa, N. B., Rajaković-Ognjanović, V. N., Jovanović, B. M., Rajaković, L. V. Determination of inorganic arsenic species in natural waters - Benefits of separation and preconcentration on ion exchange and hybrid resins. Analytica Chimica Acta 2010. Vol. 673(2), P. 185-193. doi:10.1016/j. aca.2010.05.027

25. Рунов В.К. Степанова Т. В., Пуховская В. М., Трофимчук А. К., Кузьмин Н. М. Сорбция хлоридных комплексов платиновых металлов и золота анионообменниками на основе кремнезема. Журнал аналитической химии 1993, T. 48 (11), C. 43-49. [Runov V.K., Stepanova T. V., Pukhovskaia V.M., Trofimchuk A.K., Kuz'min N.M. Sorption of chloride complexes of platinum metals and gold by anion exchangers based on silica. Journal of Analytical Chemistry 1993, Vol. 48 (11), Р. 43-49 (In Russ.)]

26. Трофимчук А.К., Жукова Л.Н., Тряшин А.С., Брускина И.М., Чернова Н.А. Синтез и кислотно основные свойства силикагелей с привитыми молекулами N-бензоил-N'пропилтиомочевины. Доклады АН УССР. Серия Б. Геол. химия и биол. науки 1989. 11, С. 5153. [Trofimchuk A.K., Zhukova L.N., Triashin A.S., Bruskina I. M., Chernova N.A. Synthesis and acid-base properties of silica gels with grafted N-benzoyl-N'-propylthiourea molecules. Reports of the Academy of Sciences of the Ukrainian SSR. Series B. Geol. chemistry and biol. Science 1989. 11, P. 51-53. (In Russ.)]

27. Losev V.N., Didukh-Shadrina S.L., Orobyeva A.S., Metelitsa S.I., Samoilo A.S., Zhizhaev A. M., Trofimchuk A.K. Effective separation of chromium species in technological solutions using amino-immobilized silica prior to their determination. Journal of Hazardous Materials 2021. Vol. 407 (5), 12438367. doi: 10.1016/j.jhazmat.2020.124383 